\title{
Room temperature midinfrared electroluminescence from GalnAsSbP light emitting diodes
}

\author{
A. Krier, ${ }^{\text {a) }}$ V. M. Smirnov, and P. J. Batty \\ Physics Department, Lancaster University, Lancaster LA1 4YB, United Kingdom \\ V. I. Vasil'ev, G. S. Gagis, and V. I. Kuchinskii
Ioffe Physico-Technical Institute, RAS, Politechnicheskaya 26, St.-Petersburg 194021, Russia
}

(Received 26 February 2007; accepted 26 April 2007; published online 24 May 2007)

\begin{abstract}
Room temperature electroluminescence in the midinfrared near $4 \mu \mathrm{m}$ is reported from GaInAsSbP light emitting diodes grown on GaSb by liquid phase epitaxy. Comparison of the electro- and photoluminescence revealed that light is generated on the $p$ side of the diode. The energy shift $(24 \mathrm{meV})$ is consistent with band gap narrowing and recombination via band tail states due to the Zn doping $\left(1 \times 10^{18} \mathrm{~cm}^{-3}\right)$ in the $p$ layer of the structure. The temperature dependent behavior of the luminescence and the improved emission intensity was attributed to recombination from localized states arising from electrostatic potential fluctuations due to compositional inhomogeneities in these alloys. (C) 2007 American Institute of Physics. [DOI: 10.1063/1.2741147]
\end{abstract}

The realization of high emittance light emitting diodes (LEDs) and lasers for the midinfrared $(2-5 \mu \mathrm{m})$ spectral range, which can work efficiently at room temperature, is hampered by competing nonradiative Auger recombination and intervalence band absorption (IVBA). ${ }^{1}$ This leads to poor internal efficiency in LEDs and high threshold currents in diode lasers. If suitable optoelectronic components could be made available one can envisage a wide variety of applications, including chemical process control, environmental monitoring of atmospheric pollution, and free space optical communications. One promising approach is to use the GaInAsPSb pentanary alloys because for a given value of the band gap (or lattice constant) properties such as the refractive index, spin-orbit valence band splitting $\left(\Delta_{\mathrm{so}}\right)$, or thermal expansion coefficient can be independently varied. ${ }^{2,3}$ This offers a different approach to control optical confinement, carrier leakage, IVBA, and Auger recombination. However, although GaInAsPSb alloys have been grown, there has been very little work in this area. ${ }^{4,5}$ Luminescence emission properties of broken gap GaInAsSbP/InAs isotype heterostructures and prototype light emitting diodes and photodiodes at short wavelengths $(\lambda<4 \mu \mathrm{m})$ have been reported for alloys grown by liquid phase epitaxy (LPE) lattice matched onto InAs. ${ }^{6,7}$ Recently, AlGaInAsSb alloys grown by molecular beam epitaxy have been effectively used as the barrier layers in room temperature midinfrared quantum well lasers. ${ }^{8}$ In this letter the epitaxial growth of GaInAsPSb/GaSb $p-i-n$ structures from the liquid phase and a study of their electroluminescence properties are presented.

Epitaxial growth of $\mathrm{Ga}_{1-x} \mathrm{In}_{x} \mathrm{As}_{y} \mathrm{P}_{z} \mathrm{Sb}_{l-y-z}$ single epilayers as well as $p-i-n$ homostructures in which $p$-type layers were doped with $\mathrm{Zn}\left(1 \times 10^{18} \mathrm{~cm}^{-3}\right)$ and $n$-type layers were doped with $\mathrm{Te}\left(4 \times 10^{17} \mathrm{~cm}^{-3}\right)$ was carried out from antimony-rich melts onto Ge doped $p$-type GaSb (100) substrates. The use of antimony as the solvent for LPE growth of GaSb-related alloys has some advantages ${ }^{9,10}$ compared with the more conventional techniques based on the low melting point metals, using In or Ga as solvents, namely,

\footnotetext{
${ }^{a)}$ Electronic mail: a.krier@lancaster.ac.uk
}

(1) Growth from $\mathrm{Sb}$ solution decreases the concentration of stoichiometric defects such as $V_{\mathrm{Ga}}+\mathrm{Ga}_{\mathrm{Sb}}$,

(2) etchback of the GaSb substrate is essentially decreased, leading to improved crystalline quality of the epitaxial layers and the heterostructure as a whole, and

(3) the same solvent can be used for all stages of the heterostructure epitaxy (which may also include AlGaIn$\mathrm{AsSb}$ layers), making it possible to obtain high reproducibility.

Growth melts were prepared from $6 N \mathrm{Sb}$ and $7 N$ In pure metals, while the sources of $\mathrm{Ga}$, As, and $\mathrm{P}$ were undoped polycrystalline GaSb, InAs, and InP binary compounds. Growth was implemented from supercooled melts at temperatures within the interval $585-600{ }^{\circ} \mathrm{C}$. In each case before the deposition of GaInAsPSb alloys a GaSb undoped $p$-type buffer layer was grown on the substrate.

In addition to structural characterization the subsequent investigation of the epitaxial structures included photoluminescence (PL) and electroluminescence (EL) measurements. Temperature dependent PL measurements over the range 4-300 K were carried out using an argon-ion laser (488 nm) for excitation of the sample. EL was measured in the temperature range $77-300 \mathrm{~K}$ from mesa etched homojunction $p-i$ - $n$ LED chips that were $750 \mu \mathrm{m}$ in diameter. These were prepared using standard photolithographic techniques and mounted episide up onto To-49 headers.

The thickness of the resulting epilayers was in the range $1.0-3.5 \mu \mathrm{m}$ when using a crystallization rate of $4-7 \mu \mathrm{m} / \mathrm{min}$ and a supercooling $\Delta T$ in the range $8-15^{\circ} \mathrm{C}$. Crystal perfection of the epilayers was measured using highresolution double crystal $x$-ray diffraction. The lattice mismatch $\Delta a / a$ was in the range $(1.9-4.1) \times 10^{-4}$ at room temperature and it was found that the optimum supercooling for the growth of lattice-matched epilayers with good surface quality was $\Delta T=13 \mathrm{~K}$ (critical supercooling was $20 \mathrm{~K}$ ). The composition of the $\mathrm{Ga}_{1-x} \mathrm{In}_{x} \mathrm{As}_{y} \mathrm{P}_{z} \mathrm{Sb}_{l-y-z}$ epitaxial layers was measured using energy dispersive $\mathrm{x}$-ray microanalysis. Using a liquidus composition of $X_{\mathrm{Ga}}^{l}=3.02 \times 10^{-4}, \quad X_{\mathrm{In}}^{l}=0.370$, $X_{\mathrm{As}}^{l}=0.024, X_{\mathrm{Sb}}^{l}=0.605$, and $X_{\mathrm{P}}^{l}=8.09 \times 10^{-4} \quad\left(T_{L}=600^{\circ} \mathrm{C}\right)$ 


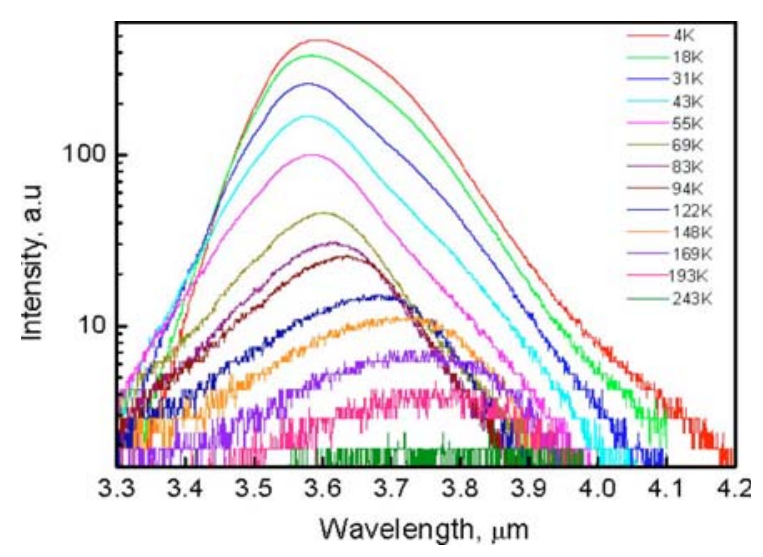

FIG. 1. (Color online) Temperature dependent PL spectra of $\mathrm{Ga}_{0.03} \mathrm{In}_{0.97} \mathrm{As}_{0.84} \mathrm{P}_{0.05} \mathrm{Sb}_{0.11}$ single heteroepitaxial layer grown on $\mathrm{GaSb}$ substrate by LPE.

at $590{ }^{\circ} \mathrm{C}$ produced a flat epitaxial layer which had a mirrorlike surface with a solid composition of $\mathrm{Ga}_{0.03} \mathrm{In}_{0.97} \mathrm{As}_{0.84} \mathrm{P}_{0.05} \mathrm{Sb}_{0.13}$. In the homojunction $p$ - $i$ - $n$ structures the composition of all the constituent epilayers was found to be identical. For selected samples secondary ion mass spectrometry was used. The resulting depth profile of a single GaInAsPSb epilayer grown on GaSb revealed that the heterointerface between the substrate and epilayer was abrupt. This confirms that interphase redistribution of components across the melt-substrate interface was absent during the epitaxial growth process.

The PL emission spectra from a single undoped epitaxial layer measured over a range of temperatures are shown in Fig. 1. The emission was broad with the principal peak shifting to the blue before following the more usual redshift associated with band gap narrowing. The integrated PL was more intense (typically $\times 3$ ) and exhibited superior temperature quenching behavior compared with other InAs and InAsSb alloys with similar band gap values. ${ }^{11}$ The temperature dependence of the band gap and the PL intensity followed closely that obtained in the literature for AlGaAsSb quaternary alloys containing compositional inhomogeneity due to phase separation. ${ }^{12}$ In this case variation in the alloy composition produces fluctuation of the electrostatic potential and results in radiative transitions from localized states near the band edges. The competition between band-band recombination and that from the localized levels leads to the observed spectral behavior.

Homojunction $p-i-n$ GaInAsPSb LED structures were fabricated and the electroluminescence emission spectra measured from one of these diodes are shown in Fig. 2. A bright EL signal was obtained over the temperature range $77-300 \mathrm{~K}$ in forward bias. A single broad peak at $305 \mathrm{meV}$ was observed at $300 \mathrm{~K}$. The atmospheric absorption of $\mathrm{CO}_{2}$ in the optical path near $4.3 \mu \mathrm{m}$ is clearly evident in the spectra. The integrated emission intensity was greater than that obtained from InAsSb LEDs with similar band gap values grown on GaSb made previously in our laboratory. ${ }^{13}$ The EL spectrum is compared with the PL spectrum obtained from an undoped GaInAsPSb epilayer in Fig. 3. The PL peak is obtained at higher energy and indicates that the EL emission originates from the $p$-region of the $p-i-n$ diode due to predominantly electron injection arising from the higher mobility of electrons compared with holes in these materials. The energy shift $(24 \mathrm{meV})$ is consistent with band gap narrowing
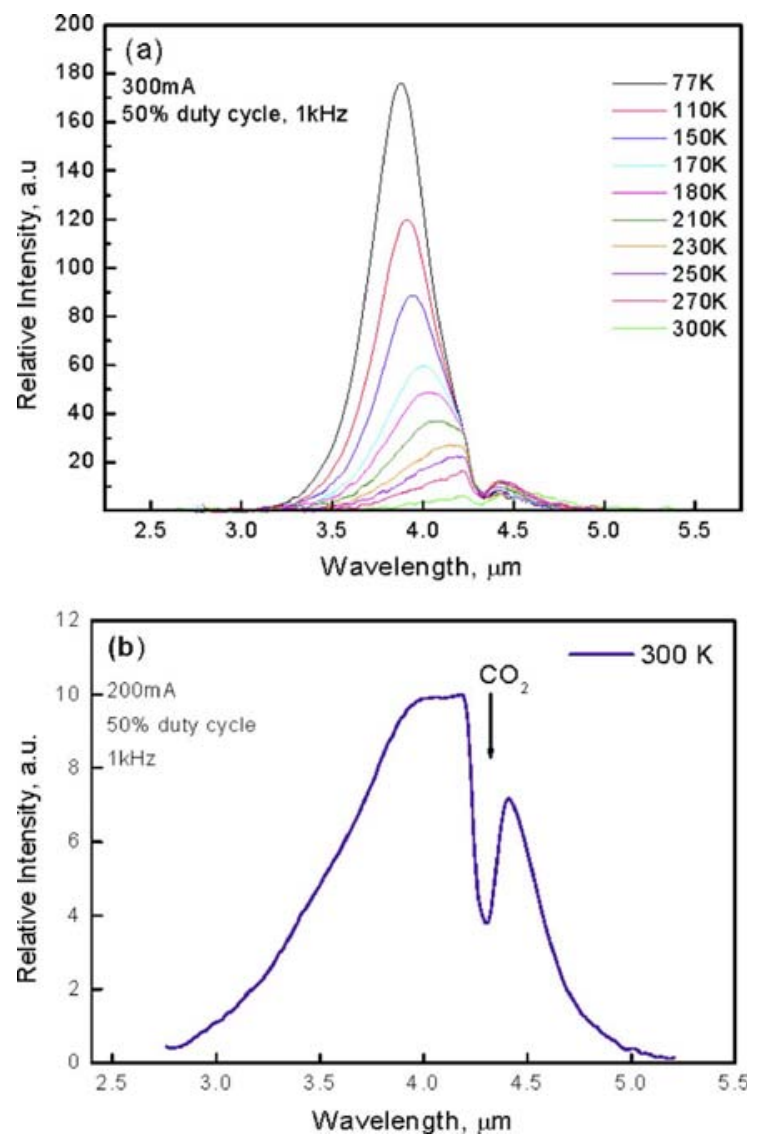

FIG. 2. (Color online) (a) EL spectra of homojunction $p-i-n$ GaInAsPSb/p-GaSb LED at different temperatures and (b) $300 \mathrm{~K} \mathrm{EL}$ emission.

associated with recombination via band tail states due to the $\mathrm{Zn}$ doping $\left(1 \times 10^{18} \mathrm{~cm}^{-3}\right)$ in the $p$ layer of the $p$ - $i$-n structure. ${ }^{14}$ We note that the reproducibility of the epitaxial growth was sufficiently good so that the compositional fluctuation between different growth runs was small (typically within $1 \%$ ) and consequently cannot account for the difference in band gap energy between PL and EL samples.

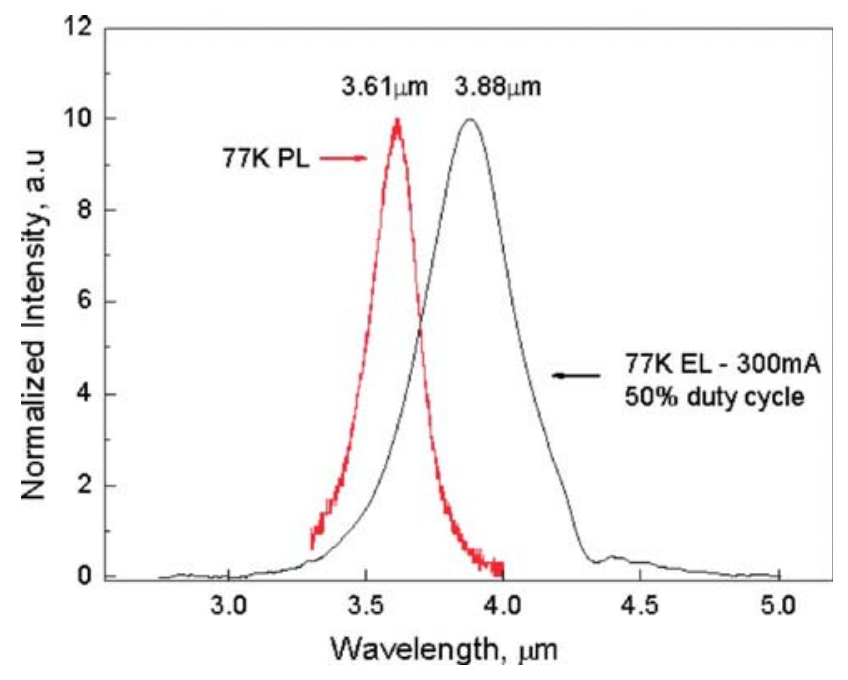

FIG. 3. (Color online) Comparison of PL from an undoped epilayer and EL from a homojunction $p-i-n$ GaInAsPSb/p-GaSb LED at $77 \mathrm{~K}$.

Downloaded 11 Mar 2008 to 194.80.32.9. Redistribution subject to AlP license or copyright; see http://apl.aip.org/apl/copyright.jsp 
A study of the electroluminescence and the light-current characteristic revealed that the recombination is dominated by radiative transitions at room temperature which is consistent with the PL behavior.

Analysis shows that the addition of phosphorus to the $\mathrm{Ga}_{1-x} \mathrm{In}_{x} \mathrm{As}_{y} \mathrm{P}_{z} \mathrm{Sb}_{l-y-z}$ alloy increases the spin-orbit splitting $\left(\Delta_{\text {so }}\right)$ and when the phosphorus composition $z$ is in the range $0.02 \leqslant z \leqslant 0.10, \Delta_{\text {so }}$ is greater than the band gap $E_{g}$. In this case, suppression of non-radiative Auger mechanism involving the spin orbit split off band takes place. ${ }^{15}$ However, in both our PL and EL experiments we estimate that the carrier excitation density is approximately $10^{16} \mathrm{~cm}^{-3}$. In this case Auger recombination is unlikely to be important and we consider that the dominant mechanism is radiative recombination. We attribute the temperature dependence of the PL and the improved electroluminescence to recombination from localized states arising from electrostatic potential fluctuations due to compositional inhomogeneities in these alloys. ${ }^{12}$ With increasing temperature these transitions give way to free-tobound transitions. We associate the localization with spinodal decomposition of the pentanary alloy, even though the surfaces of the epilayers were featureless when examined using the Nomarski microscope and x-ray diffraction spectra also revealed no evidence of compositional inhomogeneities. Our findings are in good agreement with those for AlGaAsSb lattice matched to $\mathrm{InP}$ where compositional modulation $(\sim 15 \mathrm{~nm})$ could only be observed using high-resolution transmission electron microscopy. ${ }^{16}$ The presence of spinodal decomposition in these alloys may cause concern regarding whether they are suitable for practical midinfrared optoelectronic devices. However, despite the existence of compositional modulation it is reassuring to note that devices with appropriate performance are routinely fabricated from InGaAs and InGaAsP. ${ }^{17,18}$ The low bulk diffusivity in these metastable alloys renders them stable against any further spinodal decomposition.

In summary, we have demonstrated the LPE growth of pentanary alloys lattice matched onto GaSb substrates. The epitaxial layers have mirrorlike surfaces and exhibit bright photoluminescence. Light emitting diodes based on homojunction $p-i$ - $n \mathrm{Ga}_{0.02} \mathrm{In}_{0.98} \mathrm{As}_{0.84} \mathrm{P}_{0.02} \mathrm{Sb}_{0.14}$ structures were fabricated and electroluminescence was obtained peaking near $4 \mu \mathrm{m}$ at room temperature.

The authors wish to thank EPSRC for supporting this research (GR/S75826/01) and for providing the award of a studentship for one of the authors (P.J.).

${ }^{1}$ M. Aidaraliev, N. V. Zotova, S. A. Karandashev, B. A. Matveev, M. A. Remennyi, N. M. Stus', G. N. Talalakin, and V. V. Shustov, Semiconductors 36, 1001 (2002).

${ }^{2}$ V. A. Mishournyi, F. de Anda, A. Y. Gorbachev, and V. I. Vasil'ev, J. Electron. Mater. 28, 959 (1999).

${ }^{3}$ V. I. Vasil'ev, M. V. Baidakova, E. A. Kognovitskaya, V. I. Kuchinskii, I. P. Nikitina, and V. M. Smirnov, Third International Conference on Midinfrared Optoelectronics Materials and Devices (RWTH, Aachen, 1999), p. 85 .

${ }^{4}$ V. I. Vasil'ev, I. P. Nikitina, D. Akhmedov, V. M. Smirnov, and D. A. Vasukov, Proceedings of the 27th International Symposium on Compound Semiconductors (IEEE, Piscataway, NJ, 2001), p. 233.

${ }^{5}$ V. I. Vasil'ev, I. P. Nikitina, V. M. Smirnov, and D. N. Tretyakov, Mater. Sci. Eng., B 66, 67 (1999).

${ }^{6}$ M. Aidaraliev, N. V. Zotova, S. A. Karandashev, B. A. Matveev, M. A. Remennyi, N. M. Stus', G. N. Talalakin, V. V. Shustov, V. V. Kuznetsov, and E. A. Kognovitskaya, Semiconductors 36, 944 (2002).

${ }^{7}$ M. Aidaraliev, G. G. Zegrya, N. V. Zotova, S. A. Karandashev, B. A. Matveev, N. M. Stus', and G. N. Talalakin, Semiconductors 26, 138 (1992).

${ }^{8}$ M. Grau, C. Lin, O. Dier, C. Lauer, and M.-C. Amann, Appl. Phys. Lett. 87, 241104 (2005).

${ }^{9}$ V. I. Vasil'ev, V. V. Kuznetzov, V. A. Mishournyi, V. V. Sazonov, and N. N. Faleev, Proceedings of the First International Conference on Epitaxial Crystal Growth (Trans Tech, Lausanne, 1991), Vol. 2, p. 659.

${ }^{10}$ A. G. Deryagin, N. N. Faleev, V. M. Smirnov, G. S. Sokolovskii, and V. I. Vasil'ev, IEE Proc.: Optoelectron. 144, 438 (1997).

${ }^{11}$ K. D. Moiseev, A. Krier, and Y. P. Yakovlev, J. Electron. Mater. 33, 867 (2004).

${ }^{12}$ D. O. Toginho Filho, I. F. L. Dias, E. Laureto, J. L. Duarte, S. A. Laurenco, L. C. Pocas, S. S. Prabhu, and J. Klem, J. Appl. Phys. 97, 123702 (2005).

${ }^{13}$ Y. Mao and A. Krier, Opt. Mater. (Amsterdam, Neth.) 6, 55 (1996).

${ }^{14}$ G. Borghs, K. Bhattacharyya, K. Deneffe, P. Van Mieghem, and R. Mertens, J. Appl. Phys. 66, 4381 (1989).

${ }^{15}$ J. T. Olesberg and M. E. Flatte, in Midinfrared Semiconductor Optoelectronics, edited by A. Krier (Springer, London, 2006), Chap. 1, p. 3.

${ }^{16}$ T. H. Chiu, W. T. Tsang, S. N. G. Chu, J. Shah, and J. A. Ditzenberger, Appl. Phys. Lett. 46, 408 (1985).

${ }^{17}$ F. Flas, M. J. Treacy, M. Quillec, and H. Launois, J. Phys. (Paris) 43, C5-11 (1982).

${ }^{18}$ I. T. Yamamoto, K. Sakai, and S. Akiba, IEEE J. Quantum Electron. QE-15, 684 (1979). 